\title{
Surface Instability of Liquid Propellant under Vertical Oscillatory Forcing
}

\author{
H. Q. Yang \\ CFD Research Corp./Jacob ESTS Group \\ and \\ John Peugeot ${ }^{2}$ \\ ER42, NASA MSFC
}

\begin{abstract}
Fluid motion in a fuel tank produced during thrust oscillations can circulate sub-cooled hydrogen near the liquid-vapor interface resulting in increased condensation and ullage pressure collapse. The first objective of this study is to validate the capabilities of a Computational Fluid Dynamics (CFD) tool, CFD-ACE+, in modeling the fundamental interface transition physics occurring at the propellant surface. The second objective is to use the tool to assess the effects of thrust oscillations on surface dynamics. Our technical approach is to first verify the CFD code against known theoretical solutions, and then validate against existing experiments for small scale tanks and a range of transition regimes. A 2D axisymmetric, multi-phase model of gases, liquids, and solids is used to verify that CFD-ACE+ is capable of modeling fluid-structure interaction and system resonance in a typical thrust oscillation environment. Then, the 3D mode is studied with an assumed oscillatory body force to simulate the thrust oscillating effect. The study showed that CFD modeling can capture all of the transition physics from solid body motion to standing surface wave and to droplet ejection from liquid-gas interface. Unlike the analytical solutions established during the 1960's, CFD modeling is not limited to the small amplitude regime. It can extend solutions to the nonlinear regime to determine the amplitude of surface waves after the onset of instability. The present simulation also demonstrated consistent trends from numerical experiments through variation of physical properties from low viscous fluid to high viscous fluids, and through variation of geometry and input forcing functions. A comparison of surface wave patterns under various forcing frequencies and amplitudes showed good agreement with experimental observations. It is concluded that thrust oscillations can cause droplet formation at the interface, which results in increased surface area and enhanced heat transfer between the liquid and gas phases as the ejected droplets travel well into the warmer gas region.
\end{abstract}

\section{Nomenclature}

LH2 = Liquid Hydrogen

LOX $\quad=$ Liquid Oxygen

\section{Introduction}

$\mathrm{T}$ hrust oscillation of a solid rocket motor, also called resonant burning, is a phenomenon characterized by increased acceleration pulses that may be felt during the latter stages of first-stage powered flight. These

${ }^{1}$ Chief Scientist/Research, 215 Wynn Drive, Huntsville, AL 35805, Senior AIAA Member.

2 Aerospace Engineers, Fluid Dynamics Branch-ER42, George C. Marshall Space Flight Center, MSFC, AL 35812, AIAA Member. 
longitudinal forces may increase the loads experienced by the Ares I vehicle during flight, and may exceed allowable loads on various portions of the vehicle and on the crew. Fluid motion induced during thrust oscillation can circulate sub-cooled hydrogen near the liquid vapor interface resulting in increased condensation of ullage gas and corresponding pressure collapse. Conversely, liquid contact on hot tank walls caused by sloshing can result in rapid vaporization and subsequent rapid pressure rise. Understanding the dynamic and thermodynamic behavior and the response of cryogenic propellants of Liquid Hydrogen (LH2) and Liquid Oxygen (LOX) tanks under spacecraft flight operations (such as engine shut-down and restart, oscillatory thrust and oscillatory side forces) is extremely important during the vehicle design process. In addition, the proper orientation of propellant affects the performance of several systems including the tank venting system to ensure sufficient liquid engine start-up, feed system thermal conditioning, and stability conditions in feed lines prior to $2^{\text {nd }}$ stage ignition. The orientation control of the liquid surface is also crucial to minimize vehicle disturbances and avoid excessive demands on the attitude control system [1].

The liquid surface instability of a propellant tank due to thrust oscillation can be investigated through several different approaches:

Analytical Solutions: A representative example of an analytical solution is the well-known Mathieu's Equation [2] for a cylindrical tank filled with liquid and subjected to a vertical oscillatory driving force. The solution provides important insight into the physics and dependencies on various variables. However, these solutions are only obtainable under restricted conditions and for simple geometries. Mathieu's solution also applies only to the identification of instability conditions without providing any further insight into the dynamics after the onset of the instability.

Experimental Testing: Experiments provide the most reliable and realistic information. However, experiments can be expensive and time consuming. This is especially true for full scale cryogenic testing. Normally, only small scale model and room temperature data are available. It is difficult to obtain measurements for realistic flight conditions. Only limited data (if any) exist for cryogenic fluids under thrust oscillation during flight.

Computational Modeling: The computational modeling approach of discretizing and numerically solving a relevant set of fluid and solid physics laws (the multi-physics CFD approach) has no geometrical limitation and can be applied to realistic full scale propellant tank geometries under flight conditions. It is not limited to the small amplitude regime, and can extend beyond the analytical solutions to the nonlinear regime to determine the amplitude of surface waves after the onset of instability. This is significant in that the phenomena such as droplet formation and ejection from the surface, which are due to nonlinear instability, are important drivers leading to potential ullage collapse. Computational modeling can also be applied to a range of fluids such as water, LH2, LOX, or Helium. It can bridge the analytical solutions and experiments and become a powerful design tool after systematic validation.

The purpose of this study is to investigate if a Computational Fluid Dynamics tool is capable of accurately modeling the typical transition phenomena acting on the propellant surface observed in experiments: transitioning from solid body motion to standing surface waves to droplets under increased forcing. We also assess how CFD can become a reliable tool in assisting in the design and planning of an integrated sloshing test proposed at NASA MSFC facilities. Our technical approach is to first verify a CFD program, CFD-ACE+, against known theoretical solutions, and then validate existing experiments for small scale tanks across a range of transition regimes. The verified and validated code is then applied to full scale propellant tanks to study the wave instabilities under design thrust oscillation conditions.

\section{A. Computational Modeling Tool}

The computational software used to study the tank vertical sloshing phenomenon is the commercially available CFD-ACE+ program, which was originally developed by CFD Research Corporation (CFDRC), and is currently owned and distributed by ESI [3]. CFD-ACE+ is a multi-physics and multi-disciplinary simulation tool, and is especially suited for vertical sloshing modeling. As thrust oscillation is a phenomenon of structure vibration induced by resonant burning, we will first consider the structural vibration coupled with the gas-liquid interface dynamics. CFD-ACE+ has a built-in coupled fluid-structure interaction module, and can be used to study the resonance of a tank-spring system.

CFD-ACE+ solves the Navier-Stokes equations in a Lagrangian-Eulerian frame. The continuity and momentum equation can be generally written as:

$$
\frac{\mathrm{d}}{\mathrm{dt}} \int_{\forall} \rho \mathrm{d} \forall+\int_{\mathrm{s}}\left(\rho\left(\mathrm{v}-\mathrm{v}_{\mathrm{g}}\right) \cdot \mathrm{ds}\right)=0
$$

2

American Institute of Aeronautics and Astronautics 


$$
\frac{\mathrm{d}}{\mathrm{dt}} \int_{\forall} \rho \phi \mathrm{d} \forall+\int_{\mathrm{s}} \rho \phi\left(\mathrm{v}-\mathrm{v}_{\mathrm{g}}\right) \cdot \mathrm{ds}=\int_{\mathrm{s}} \mathrm{q} \cdot \mathrm{ds}+\int_{\forall} \mathrm{S}_{\phi} \mathrm{d} \forall
$$

where $\phi$ are the Cartesian velocity components, $v$ is the absolute fluid velocity, $q$ the diffusive flux and $S_{\phi}$ the volume sources. $\forall$ is the computational cell volume and $\mathrm{S}$ are bounding cell surfaces, $\mathrm{v}_{\mathrm{g}}$ is the grid velocity. As the grid is moving with time for the present fluid-structure interaction problem, a space conservation law (SCL) is enforced during the grid deformation,

$$
\frac{\mathrm{d}}{\mathrm{dt}} \int_{\forall} \mathrm{d} \forall=\int \mathrm{v}_{\mathrm{g}} \mathrm{ds}
$$

CFD-ACE+ also features a structural dynamics module, FEMSTRESS. Its main capabilities include:

- triangular, quad, tetrahedral, prisms, or brick elements;

- linear or high-order isoparametric elements;

- small or large deformation;

- elastic or plastic stress;

- isotropic or anisotropic materials;

- thin to thick shell/plate elements;

- modal analysis and eigenvalue solutions; and

- steady and dynamic analysis.

FEMSTRESS uses the finite element method and solves the equation of motion:

$$
[\mathrm{M}]\{\ddot{\mathrm{q}}\}+[\mathrm{C}]\{\dot{\mathrm{q}}\}+[\mathrm{K}]\{\mathrm{q}\}=\{\mathrm{F}\}
$$

where $\{\mathrm{q}\}$ is the displacement vector, $[\mathrm{M}]$ is mass matrix, $[\mathrm{C}]$ is the damping matrix, $[\mathrm{K}]$ is the stiffness matrix, and $\{\mathrm{F}\}$ is the force vector due to the fluid dynamic load and shear stresses.

FEMSTRESS supports several boundary conditions specified from a Graphic User Interface (GUI):

- specified load: pressure or concentration loads;

- coupled pressure load from fluid;

- specified body forces;

- specified displacements: zero (fixed) or prescribed motion;

- symmetry conditions; and

- contact condition: contacting surfaces and target surfaces for elastic-rigid contact, and, elastic-elastic contact.

Related to the present free surface, CFD-ACE+ contains a Volume of Fluid (VOF) module which is designed for applications involving two immiscible fluids. In the current application, the first fluid is liquid LH2, LO2, or water, and the second fluid is gaseous $\mathrm{H} 2, \mathrm{O} 2$, helium or water vapor, respectively. In the VOF module, a single set of momentum and continuity equations is solved, but different property sets are defined for each fluid. The volume fraction of one phase (in this case the liquid phase) is tracked throughout the solution to determine which fluid occupies each computational cell at any given time. In cells containing both fluids, a special routine is used to locate the shape, location and normal of the interface. When there is a surface tension force, its effect is applied in a conservative form [4]. For time dependent simulations such as the present tank under oscillatory force, a special second order algorithm is used to update the volume fraction in a cell from one time step to the next. The second order geometric reconstruction scheme for the interface representation is employed to track the interface. This unique reconstruction algorithm is currently available only for structured grid. It is due to this algorithm that the present CFD solution is capable of capturing and maintaining a sharp interface between the phases for very long period of time. The time step size is determined by the local Courant-Friedrichs-Lewy (CFL) number. Some of the validation studies and applications to ARES I vertical and side sloshing were presented in a previous JANNAF paper [5]. The validation study for the tank sloshing is reported in reference [6].

\section{Results And Discussion}

Modeling vertical sloshing under thrust oscillation is a challenging task. It involves structural dynamics resonance, fluid-structure coupling, and gas-liquid interface dynamics. The structural dynamics equations are usually written in the Lagrangian frame, whereas the fluid dynamics equations are in the Eulerian frame. Some type of moving mesh has to be invoked to match the moving structure and fluid grid at the structure-fluid interface. This study will take a building block approach: building up to the final complete 3D propellant tank model advancing through a series of simple, reduced dimensionality elemental verification and validation cases. First, a 
2D axisymmetric, multi-phase model of gases, liquids, and solid structures is used to verify that CFD-ACE+ is capable of modeling fluid-structure interaction and system resonance. The 2D model gives fast turn around time and builds confidence in the accuracy of the final full 3D fluid-structure and VOF interaction study. Then, the 3D mode will be studied with the assumption of oscillatory body force to simulate the thrust oscillating effect.

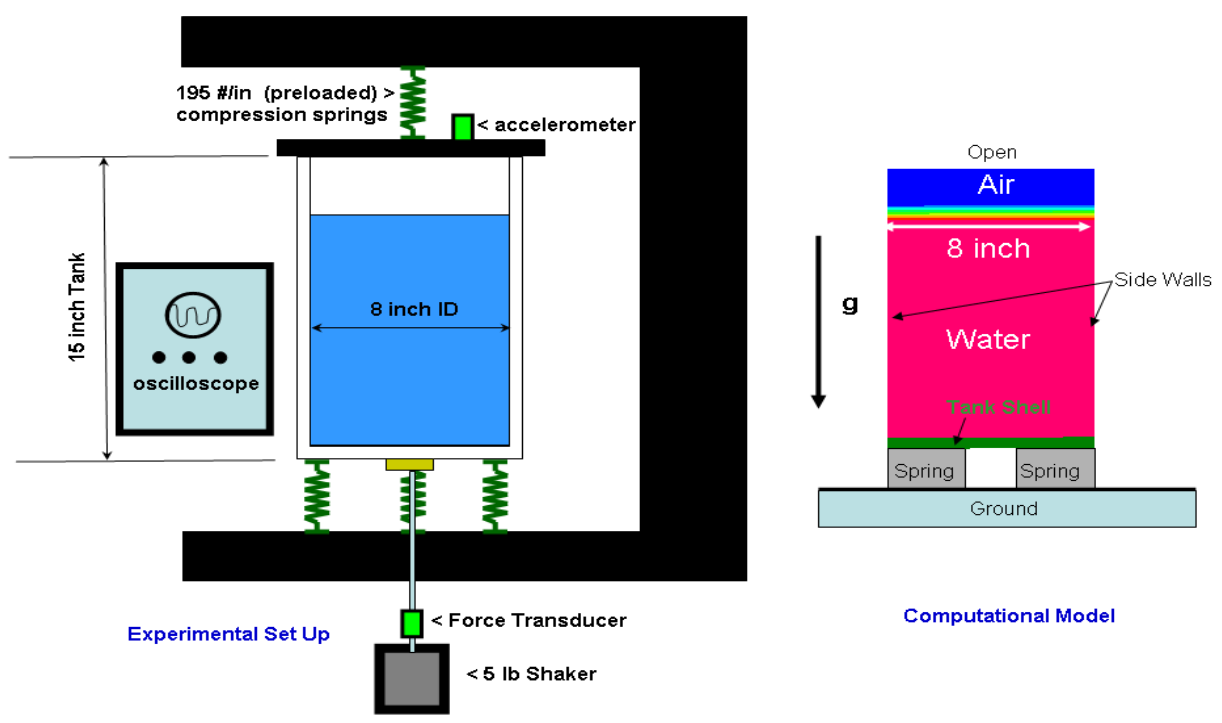

Figure 1. Experimental Setup and Simulation Model of the Tank and Elastic Structure

\section{A. 2D Self-Vibration Fluid-Structure Interaction Model}

The basic experimental set up at NASA MSFC [7] and the computational model are described in Figure 1. The tank has an 8" diameter and 15" height, with a 75\% initial water fill level, and is supported by elastic springs. A shaker acts at the bottom of the tank to provide the driving force for the "thrust oscillations". The maximum force from the shaker is rather small, but it can cause thrust oscillations when resonating with the system. The natural frequency of the system is primarily determined by the elastic stiffness of the springs and the mass of water in the tank. The computational model simulating the spring-tank interaction and free surface dynamics is presented in Figure 1. Here the bottom wall of the tank is modeled as an elastic solid along with the springs, and the side wall is treated as rigid without mass. The interface between the liquid and bottom wall is treated as a fluid-structure interface, where the continuity of displacement, velocity, normal stresses and shear stresses from the fluid side and the solid side are satisfied at all times. The fluid dynamics is modeled using Finite Volume Method (FVM) and the free surface is tracked by VOF. The springs are modeled as elastic structures, and are calculated by Finite Element Method (FEM). The computational grid and boundary conditions are shown in Figure 2.

\section{B. Self-Vibration Modeling}

First, the self-vibration of the system is studied by initially setting the spring at an unstressed state. At $t=0$, the gravitational force of the tank is applied to the spring, so that the self vibration starts. Since the spring is modeled as a solid block, the equivalent Young's modulus has to be determined. In this study, the value of Young's modulus is selected such that the system will resonate at $15 \mathrm{~Hz}$, the same resonant frequency as in the experiment. Figure 2 shows the time history of the vertical displacement at a monitor point inside the solid block. During a one second time frame, the spring displacement shows 15 cycles, giving an oscillating rate of $15 \mathrm{~Hz}$. Due to fluid dissipation, the amplitude of the oscillation decays, and eventually, the oscillation stops, when the spring force balances the mass of the tank. From an animation of Figure 2, one can clearly see the spring compression and expansion as the tank moves up and down. This example demonstrates that the essential physics of coupled fluid-structure interaction and free surface dynamics involving three phases of solid, gas and liquid are well captured. 


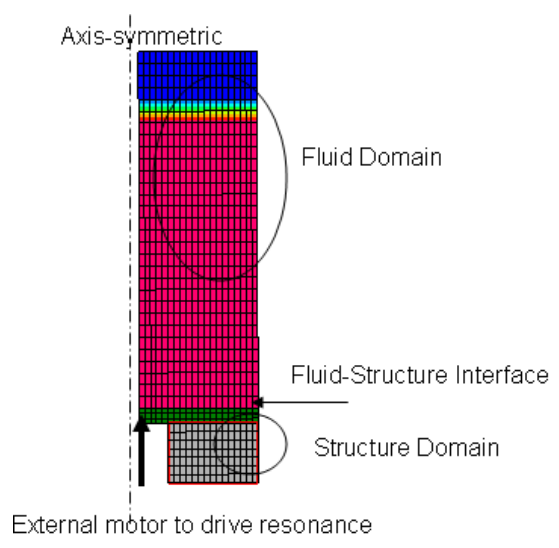

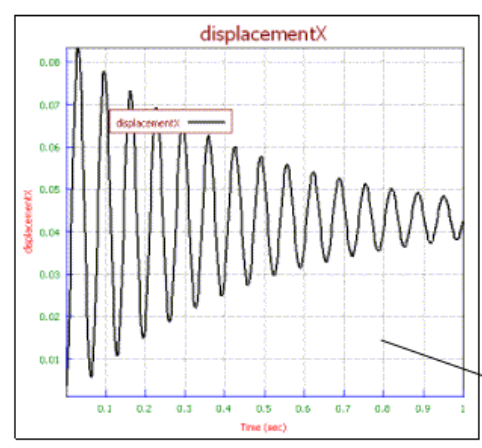

Displacement at a point in the solid (spring) in (inch)

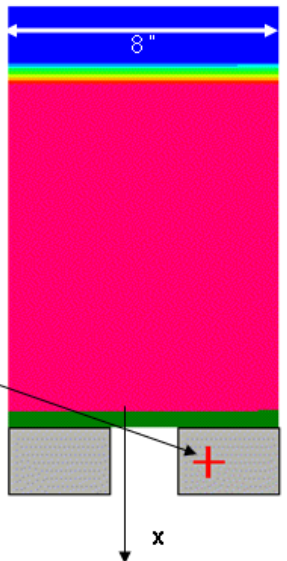

Figure 2.

Modeling of Fluid-Structure Interaction of Self-vibration of the Tank and Spring System.

\section{Forced Vibration to Simulate Thrust Oscillation.}

Next, a time dependent pressure force is applied to the bottom of the tank as shown in Figure 3. The force is oscillatory and at the natural frequency of the system of $15 \mathrm{~Hz}$. Resonance occurs, which is indicated by the increase of vibration amplitude of the spring plotted in Figure 3. The displacement history at the same location as in Figure 2 is shown in Figure 3. The amplitude increases with time, and eventually settles down as a "limit cycle", where the supplied energy is balanced by viscous dissipation. The corresponding liquid-gas interface contours in Figure 3 show small waves that grow and eventually become standing waves. The surface dynamics is the same as observed in the experiments, except that the waves are in the form of concentric rings due to the 2D limitation, instead of droplet type of peaks and valleys in 3D.

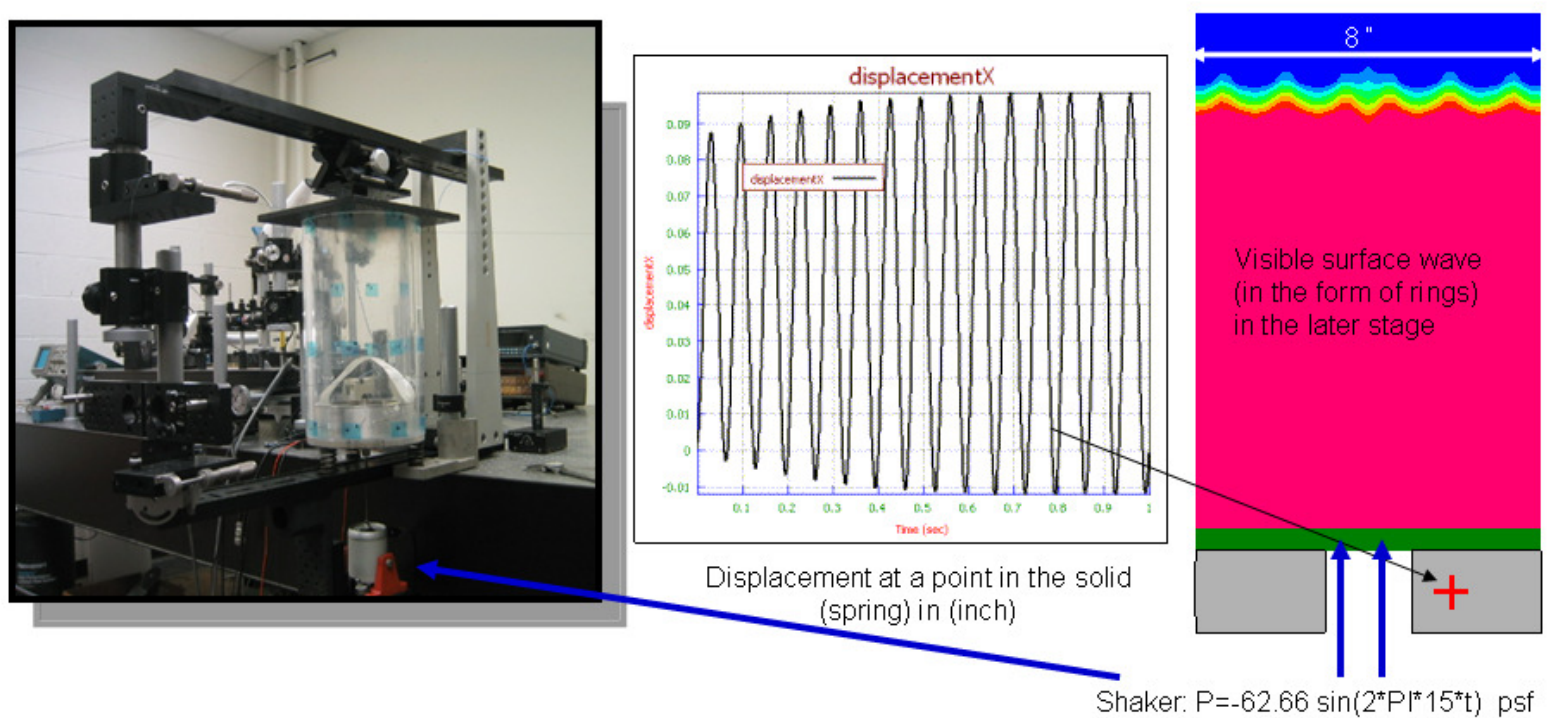

Figure 3. Fluid-Structure Interaction for Forced Vibration.

\section{3D Tank Modeling}

The above example clearly demonstrated the unique capability of CFD-ACE+ in capturing the fundamental dynamics of thrust oscillation induced surface wave, the coupled fluid-structure interaction, and multi-phases (solid, gas, and liquid) dynamics. In order to quantitatively compare with analytical solution and with experiment result, a 3D computational model was generated. To save computer resources and for fast turn around time, the Thrust Oscillation (TO) effect was modeled as a body force, such that the tank is set in a non-inertial frame which moves 
with the rigid tank wall. The fluid is assumed to be isothermal, and the physical properties are constant. The diameter of the tank is 8 ", as in the experiment, and a volume grid with a 'butterfly' structure featuring approximately 60,000 cells was generated inside the tank as illustrated in Figure 4. The initial condition of the liquid interface is shown in Figure 4, with the liquid phase marked in red and the gas phase in blue. The upper boundary is open to the ambient, and the remaining boundaries are defined as walls with zero slip velocity. The TO effect is modeled as time dependent body force with a parameter $\alpha$, which relates to the vertical body force as:

$$
g_{y}=g *(1+\alpha \sin (2 \pi f t))
$$

The value of $\alpha$ represents the $g$ level of the oscillatory acceleration in the vertical direction.
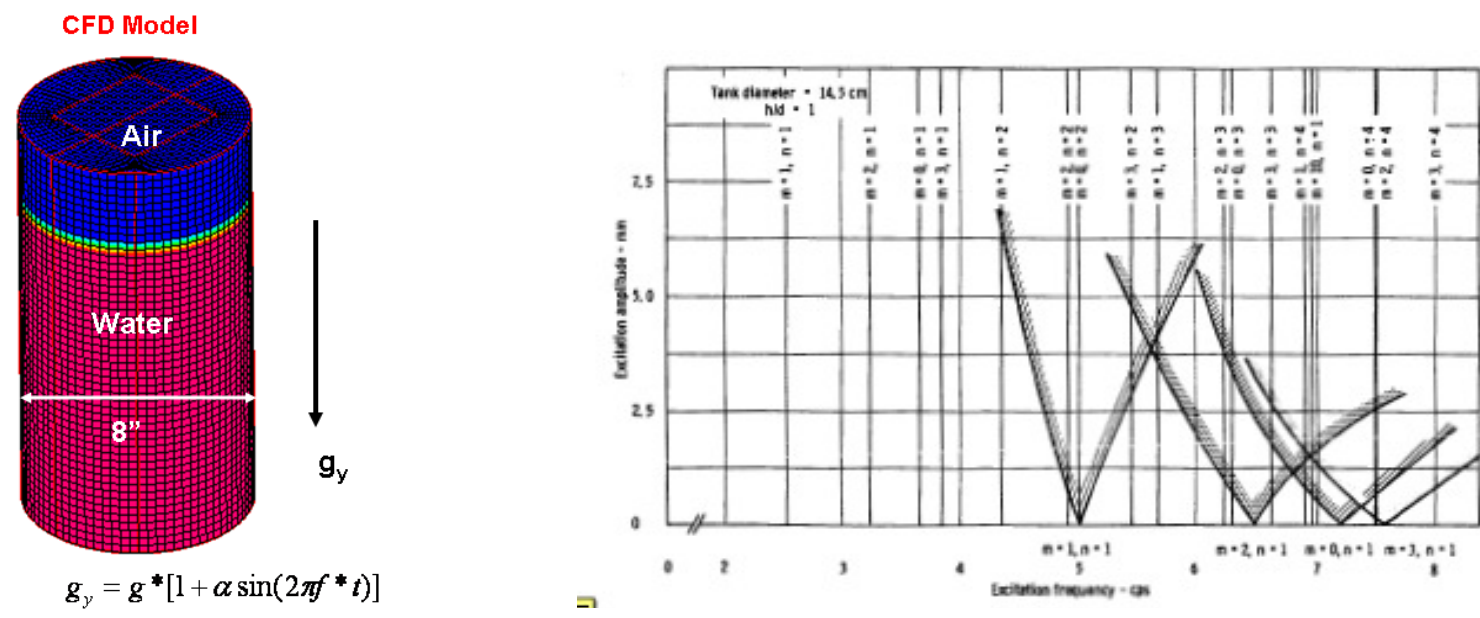

Figure 4. 3D CFD model for 8"

Figure 5. Stability charts for the solution of Mathieu's equation [2]. cylinder tank.

\section{E. Initial Linear Stability Regime.}

Natural Frequency of the Surface Wave

The interfacial dynamics of a cylindrical tank containing a heavy liquid forced to vibrate vertically has been studied in 1960's using both analytical and experimental methods. The surface dynamics is called Faraday's wave, and a comprehensive review can be found in Chapter 8 of reference [2]. With the assumption of incompressible and irrotational flow, and with neglected gas phase effects, a neutral stability equation has been derived, which is in the standard form of Mathieu's equation. The eigenmodes of Mathieu's equation give stability charts as shown in Figure 5. For each eigenmode, there is a modal frequency and a modal shape function. The modal frequency is the natural frequency of the surface wave, and the modal shape represents the free surface wave form.

One of the eigenmodes of the current 8" tank is shown in Figure 6(a) (Courtesy of Dr. Bernard Beard of ARES Corp.). The color represents the shape of the wave, with red for the peaks, and the blue for the valleys. Figure 6(a) is the mode of $(6,4)$ with eigenvalue of $14.995 \mathrm{~Hz}$, which is close to the driving frequency under the consideration. There are other modes which have natural frequency close to $15 \mathrm{~Hz}$ for the 8 " tank. 


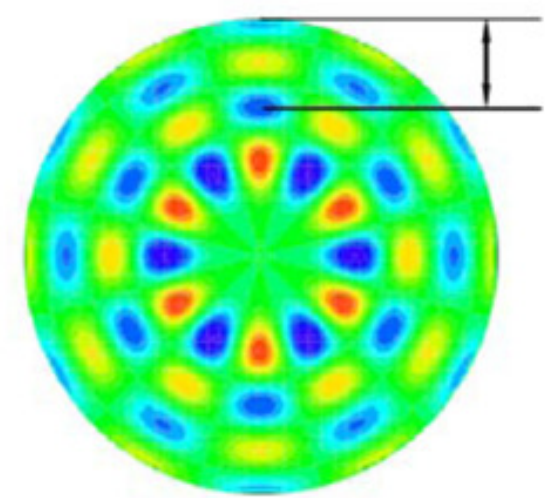

a) Analytical solution. $(6,4)$ mode shape $@ 14.995 \mathrm{HZ}$ from Mathieu equation (Courtesy of Dr. Bernard Beard of Ares Corporation)

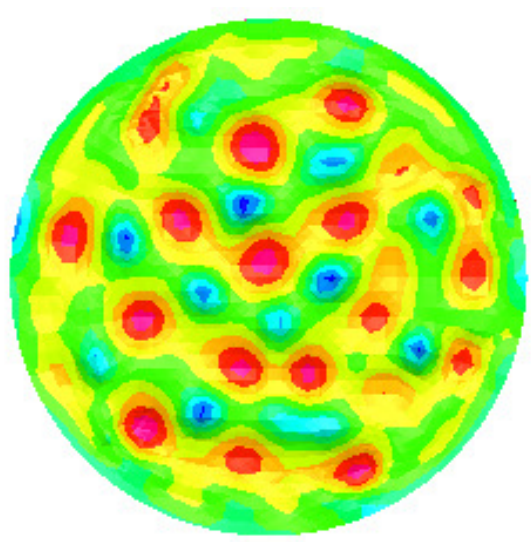

(b) Standing surface wave form from CFD-ACE Simulation. Combination of $(4,6)$ mode and $(3,7)$ mode.

Figure 6. Comparison of surface wave form and wavelength between analytical solution and CFD simulations.

\section{Surface Wave Response to the Excitation Forcing Frequency}

A simpler version of Figure 5 is shown in Figure 7(a) [2]. Here, $\omega_{n}$ represents one of the natural frequencies close to the forcing frequency. The horizontal axis represents the driving frequency parameter. The vertical axis in Figure 7(a) is the wave amplitude with the curves presenting the neutral stability. For any state above the curve, the surface wave is unstable, and for the state below the curve, the wave is stable. When the excitation frequency, which is the tank-spring resonant frequency for the current system, coincides with one of modal frequencies (frequency parameter is equal to 1.0 in Figure 7(a)), a small amplitude forcing will lead to the surface wave growth. However, when the excitation frequency does not coincide with the natural frequency of the surface wave, the free surface will become unstable only when the amplitude of the excitation is larger than a certain value.

\section{Onset Frequency and Forcing Amplitude Validation}

To study this amplitude dependent instability, numerical simulations were carried out using various $\alpha$ values from 0.1 up to 2.0 at $15 \mathrm{~Hz}$ (see Equation (5) for the definition of $\alpha$ ). All simulations started with the quiescent no motion state. The interface wave amplitudes were recorded from the CFD simulations and are shown in Figure 7(b). At smaller values of the excitation force, when the $\alpha$ value is below 0.8 , there is no sloshing motion at all. The surface of the liquid-gas is essentially flat, and the liquid column acts as a rigid body and vibrates up and down. The pressure field stratifies instantaneously with the time dependent body force variation. However, when the oscillatory acceleration $\mathrm{g}$ level $(\alpha)$ is above 0.8 , surface instability sets in, and a pattern of standing waves can be observed on the free surface. The wave amplitude is found to increase with increasing $\alpha$ as seen in Figure 7(b). This evolution of dynamics is just as predicted from the nonlinear solution of Mathieu's equation under large amplitude shown in Figure 7(a). If the driving frequency is off the natural frequency of the surface wave, only when the amplitude of the driving force is above the neutral value, will the instability set in.

\section{Wavelength and Wave Form Validation}

To compare the surface wave shape, the CFD solution is given in Figure 6(b), along with the analytical solution of the modal shape expressed in terms of Bessel functions. It should be noted that there are several natural modes for this 8" diameter tank with frequency close to $15 \mathrm{~Hz}$. Shown in Figure 6(a) is just one of these frequencies, with the mode $(6,4)$. The CFD simulation shows very similar wavelength and wave pattern, but it may well be a combination of the $(4,6)$ and $(3,7)$ modes. One should also notice that the waveform of Figure 6(b) is in large amplitude regime. Regardless, agreement between the analytical solution and CFD prediction is fairly good. To make further comparison with experiment, the photographs from the test along with surface shape from CFD simulation are given in Figure 8. Again, the agreement is good in terms of wavelength and wave form. 


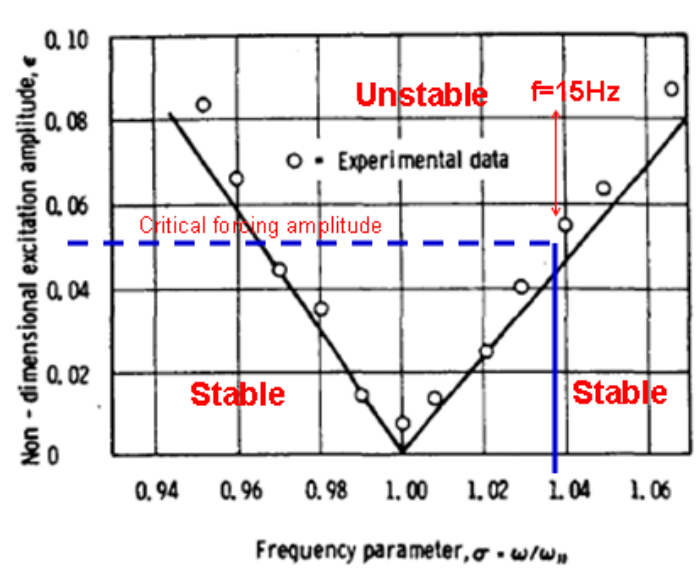

(a) Analytical Solution from Mathieu's Equation [2]

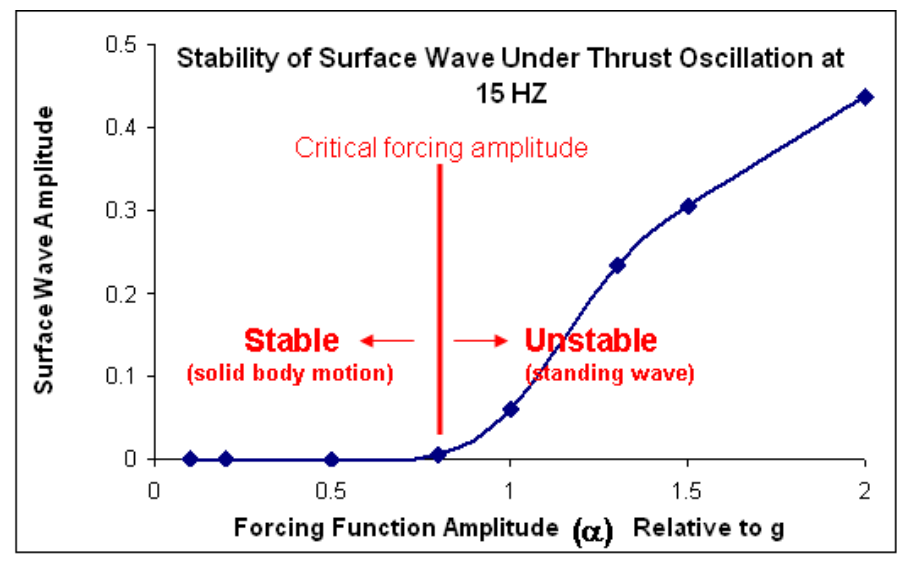

(b) Present CFD Solution Analytical Solution

Figure 7. Stability curve from CFD solution and from analytical solution.

Surface Wave Response Frequency Validation

One of the interesting features of vertical sloshing is that the wave frequency, or the response frequency of surface wave, usually occurs at exactly one-half that of the tank motion. Although in some cases the liquid frequency is equal to the forcing frequency and in other cases is greater than the forcing frequency. This is very different from side sloshing where the frequency of the free surface to the transverse excitation corresponds precisely to the forcing frequency. This apparently anomalous behavior for vertical excitation was first noticed by Faraday [2] during a series of investigations of vibrating plates covered by a thin layer of water. The liquid frequency was recorded in the present CFD simulation, and it was found that the liquid oscillation was indeed at the one-half subharmonic of the forcing motion.

Validation Summary for the Linear Stability Regime

These simulation results again show the capability of CFD in capturing the fundamental physics in terms of a) onset frequency; b) surface wave form; c) driving forcing amplitude; and d) surface wave response frequency.

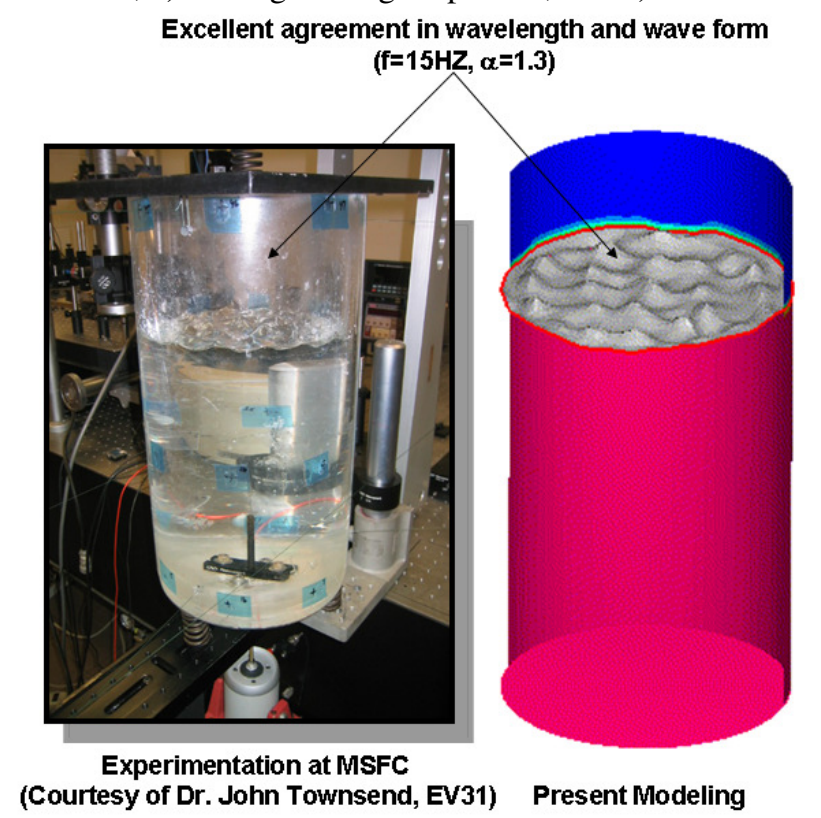

Figure 8. Comparison of surface wave form and wavelength between experiment testing and CFD simulations

8

American Institute of Aeronautics and Astronautics 


\section{F. Non-Linear Regime}

The above test case shows that CFD-ACE+ is capable of determining the transition characteristics and posttransition behavior of Faraday's waves (not possible by linear analytical solutions) for smaller amplitude of the forcing frequency, i.e. transition from solid body motion to standing wave motion. The experimental work of Goodridge et al. [8] took the above Faraday's waves further into the nonlinear regime, where the unbroken surface forms and ejects droplets under parametric excitation. They found that with increasing forcing, an initially flat surface wave state will evolve into a periodic standing wave state. This then will change into an aperiodic state, which, at sufficiently large forcing, will produce large-amplitude waves in the form of jets (or spikes) which will break into droplets. The purpose of this study is to investigate if a CFD tool can model the observed droplet ejection from the surface wave due to TO at a high amplitude of the forcing, and to determine the grid resolution requirement to resolve the droplets from the first principle.
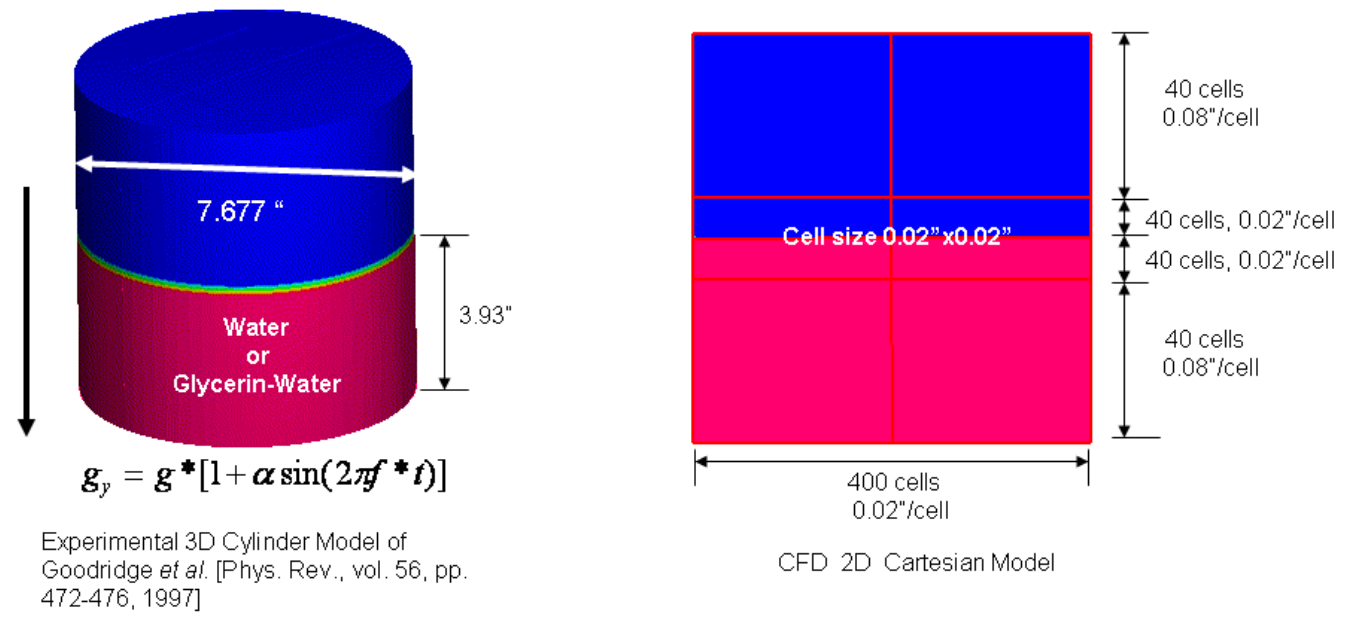

Figure 9. Experiment and simulation model to study the droplet ejection of Faraday's waves under large driving force.

The experimental configuration of Goodridge et al. [8] is shown in Figure 9, where a cylindrical plastic container of $20 \mathrm{~cm}$ (7.677") diameter with initial liquid filled to $10 \mathrm{~cm}$ (or 3.93“) is mounted on the armature of a electromagnetic shaker. The liquids used in the experiment were water, water-glycerin, and ethanol, representing different viscosities. Our study was first done using a 2D model, where 400 grid cells were spaced evenly along the horizontal direction, and variable grid sizes were used along the vertical direction with a denser mesh near the interface to ensure that sufficient grid resolution was applied to capture the high frequency small wavelength waves.

The experimental work of the Goodridge et al. [8] covered the forcing frequency from $20 \mathrm{~Hz}$ up to $80 \mathrm{~Hz}$. Under high frequency forcing, the response wavelength of the natural mode decreases with the increase in the forcing frequency. Depending on the wavelength, the restoring energy from ejection could be either surface tension or gravitational force. At smaller wavelengths, surface tension can be dominant. To determine the critical wavelength between the two forces, one can derive a dispersion relationship as shown in [10] for the twodimensional gravitational wave of Figure 10 as:

$$
\omega^{2}=g k+\frac{\sigma k^{3}}{\rho}
$$

where $\omega$ is the angular frequency, $g$ is the gravitational acceleration, $\mathrm{k}$ is the wave number, $\sigma$ is the surface tension, and $\rho$ is the liquid density. With $\mathrm{k}=2 \pi / \lambda, \lambda$ as the wavelength, one can find the frequency as:

$$
f=\sqrt{\frac{g}{2 \pi \lambda}+\frac{2 \pi \sigma}{\rho \lambda^{3}}}
$$




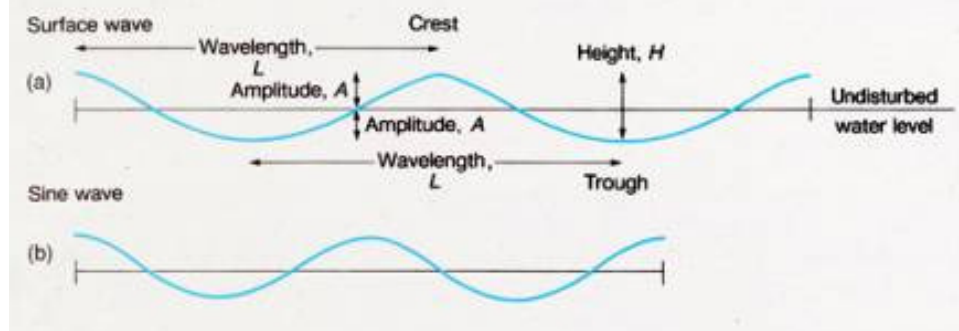

Figure 10. Surface gravitational wave on liquid and gas interface

It can be seen that the wave frequency depends on surface tension, gravity acceleration, and wavelength. A critical wavelength can be found when the surface tension contribution is equal to that of gravity:

$$
\begin{aligned}
& \frac{g}{2 \pi \lambda_{c}}=\frac{2 \pi \sigma}{\rho \lambda_{c}{ }^{3}} \\
& \lambda_{c}=2 \pi \sqrt{\frac{\sigma}{\rho g}}
\end{aligned}
$$

At ground conditions (sea-level gravity) with water properties, one can find that $\lambda_{c}=0.017 \mathrm{~m}$. Thus, when the wavelength of the free surface is less than $0.017 \mathrm{~m}$, or 0.67 ”, the surface tension force becomes the main restoring force, and capillary waves will disintegrate. Based on the critical wavelength, we can also find a critical frequency of $13.8 \mathrm{~Hz}$. When the forcing frequency is below $13.8 \mathrm{~Hz}$, the restoring force is the gravitational force, whereas when the forcing frequency is larger than $13.8 \mathrm{~Hz}$, the surface tension force becomes important. In the following discussion, In the following discussion reference will be made to a critical wavelength greater than 0.67 " and corresponding critical frequency of $13.8 \mathrm{~Hz}$.

\section{Driving Frequency at $20 \mathrm{~Hz}$}

First we consider the case when the driving frequency is at $20 \mathrm{~Hz}$. At this frequency, the resulting surface wave dynamics are dominated by the surface tension. As the resulting wavelength is below $1.7 \mathrm{~cm}$, the container boundaries (which are $7.67 \mathrm{~cm}$ apart) should have less influence on the results. Representative results are given in Figure 11. The experimentally determined threshold value of acceleration from reference [8] is shown at the lower right corner of the figure. For the case with water as the liquid, the ejection threshold of alpha is slightly larger than 1.0 from the test data.

Two simulations are shown in Figure 11, one using $\alpha$ of 1.0 and one of 2.0. For the case with $\alpha=1.0$, the standing wave is visible and there are no droplets stripped from the surface. The surface integrity is intact. The standing wave leads to a surface area increase which may potentially increase heat transfer between liquid and gas phase, but this increase is not significant. However, when the forcing amplitude increases to $\alpha=2.0$, as shown in the same figure, the surface standing waves disintegrate and form a large amount of droplets from the surface. In the animation (not shown in this paper, but in the presentation), the droplets form a dense spray, and they remerge with the liquid surface. Due to high initial break-up velocity, the droplets could enter into the region beyond the interface neighborhood. For propellant tanks, these droplets can greatly enhance heat transfer between liquid and gas phases. Not only will they carry heat away from the gas phase near the free surface due to phase change, but also they carry heat away from the warmer gas well within the ullage region. This type of droplet ejection can lead to potential ullage collapse.

An experimental photograph from Reference [8] is used for comparison with the CFD simulation in Figure 11. Again, good agreement is evident in terms of wavelength, wave pattern, and spawned droplets. Favorable comparison is also found in the threshold acceleration value shown in Figure 11, where $\alpha=1$ is below the threshold and $\alpha=2$ is above the threshold. As the droplet sizes are all smaller than 0.67 ", the surface disintegration is surface tension dominated. It should be pointed out that the smallest droplet size that can be captured in the simulation is 
about the grid cell size of 0.02 ". Objects smaller than the grid cell cannot be resolved numerically with the present phase interface tracking algorithm.

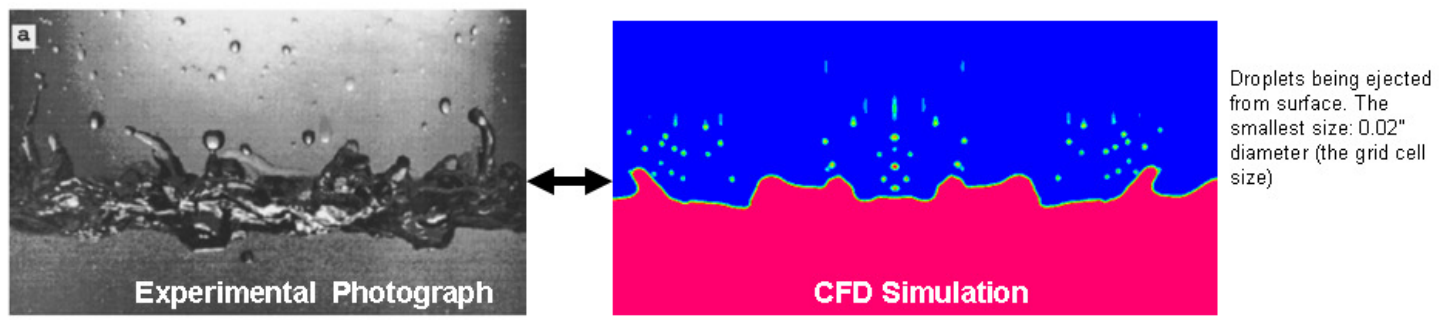

$\mathrm{f}=2 \mathrm{OHz}, \alpha=2.0$

Droplet ejection from experiment and simulation

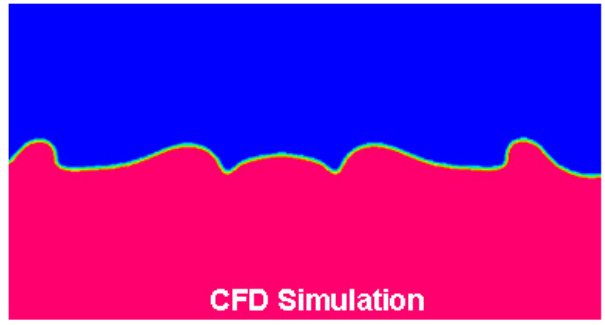

$\mathrm{f}=20 \mathrm{~Hz}, \alpha=1.0$

No droplet ejection from experiment and simulation

Good agreement between present CFD model and experiment.

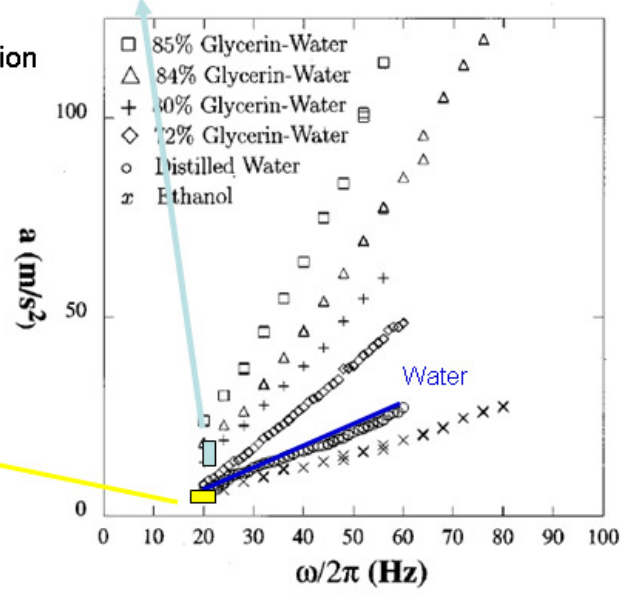

Threshold Acceleration from Goodridge et al. (1997)

\section{Figure 11. Droplet ejection modeling and comparison with experiment at $20 \mathrm{~Hz}$ using water}

\section{G. Driving Frequency at $60 \mathrm{~Hz}$}

When the forcing frequency increases, the disintegration process is suppressed at the same forcing amplitude of $\alpha=2$. This is evident in Figure 12. With $\alpha=2$, there is no droplet ejection from the surface, rather a standing wave is observed. The CFD simulation is consistent with the experimental observation, where the threshold increases with increasing forcing frequency (see the experimental curve in the lower right hand side of Figure 12). One can also see that the wavelengths have reduced in comparison with those at the case of $f=20 \mathrm{~Hz}$. However, for a large driving force, a large amount of small droplets can be seen stripped from the surface. Comparison of droplet sizes and surface wavelength between CFD and experiment is again fairly good. The threshold value is bounded from the present CFD simulations and its value is between $2<\alpha<4$. 

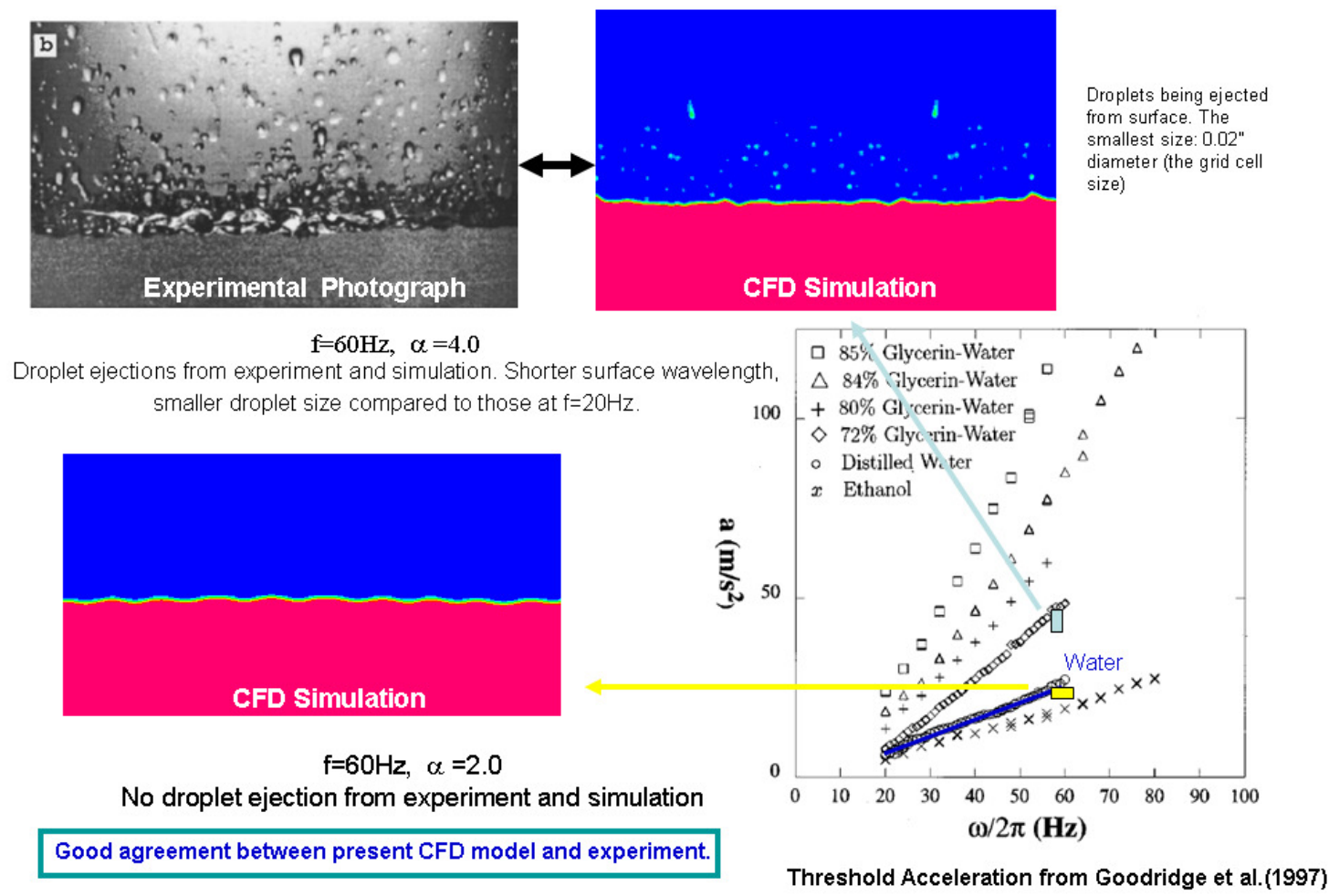

Figure 12. Droplet ejection modeling and comparison with experiment at $60 \mathrm{~Hz}$ using water

\section{H. Viscous Effects in Droplet Ejection Waves}

The above comparisons were made for air and water as the fluids. The experiments of Goodridge et al. [8] using different fluids found that low-viscosity fluids have threshold accelerations which depend on only surface tension and forcing frequency, while high viscosity fluids have thresholds which depend on only viscosity and forcing frequency. This effect is shown in Figure 11 with various fluids. In order to simulate the experimental observation, CFD simulations were made using a glycerin-water mixture with a viscosity 43 times higher than water. The simulated instantaneous surface wave and the corresponding experimental photograph are shown in Figure 13. In contrast to water where the wave breaks up into droplets immediately, here several long filament-like peaks are visible before break off occurs. The high liquid viscosity enables the peaks to achieve their filament structure. The comparison with experiment in terms of the wave structure is rather remarkable as seen from Figure 13. The higher viscosity can stabilize the droplet ejection, as shown in Figure 14, where the same driving frequency and acceleration level $\alpha=2$ are shown for water and glycerin-water. The small droplets observed in water are suppressed in glycerin-water. 


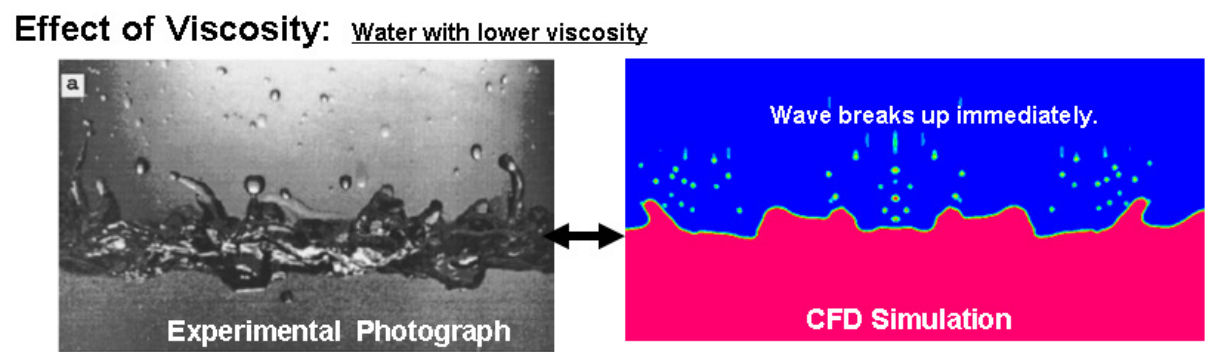

$\mathrm{f}=20 \mathrm{~Hz}, \alpha=2.0$

Effect of Viscosity: Glycerin-Water with higher viscosity

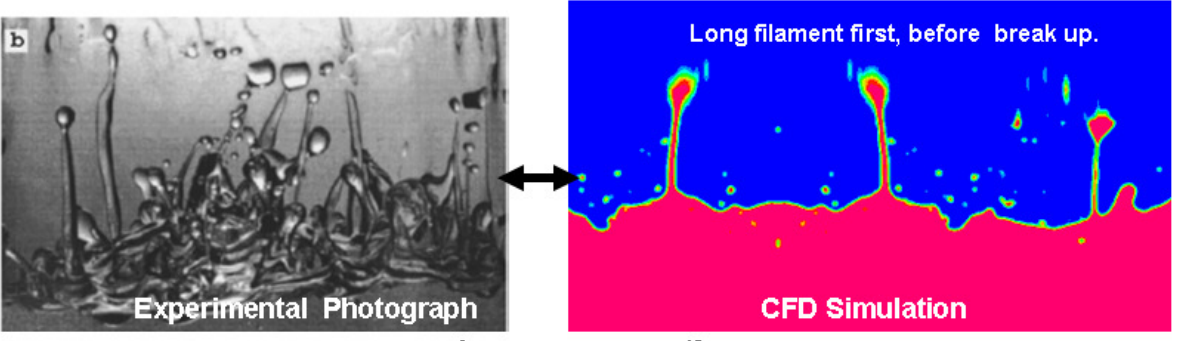

$\mathrm{f}=20 \mathrm{~Hz}, \alpha=3.0, \mu=43 \times \mu_{\text {rrater }}$

Different from water, where wave breaks up into droplets immediately. Here several long filament like peaks are visible before break off occurs.

Figure 13. Droplet ejection modeling and comparison with experiment at $60 \mathrm{~Hz}$ using Glycerin-water

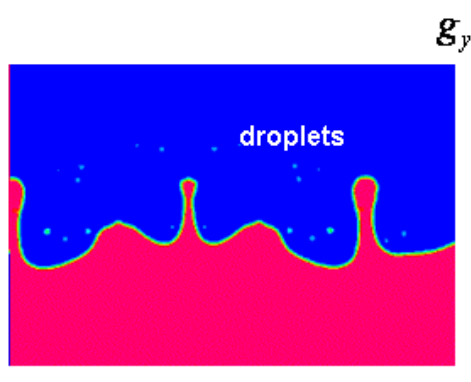

Water, $\alpha=2.0$ $g_{y}=g^{*}\left[1+\alpha \sin \left(2 \pi f^{*} t\right)\right] \quad \mathrm{f}=2 \mathrm{OHz}$

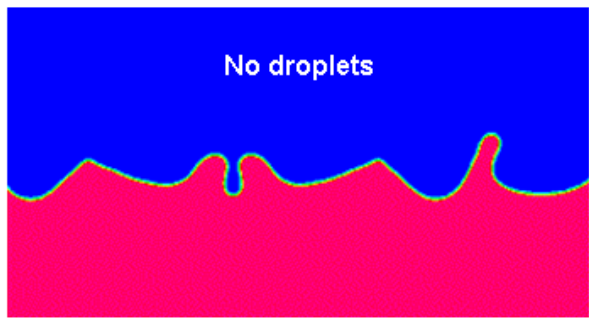

Glycerin-Water, $\alpha=2.0$

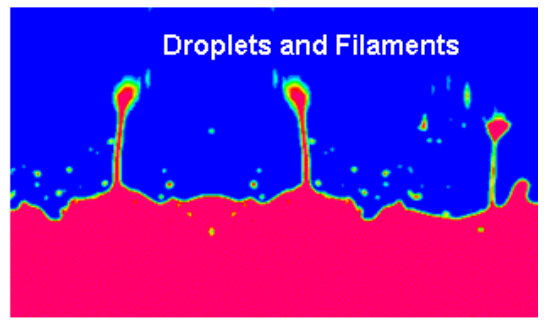

Glycerin-Water, $\boldsymbol{\alpha}=\mathbf{3 . 0}$

Figure 14. Characteristics of droplet formation at different acceleration level and for different fluids.

\section{Summary And Conclusions}

The multi-phase fluid interaction simulation capability of the CFD software program, CFD-ACE+, has been demonstrated and validated on several key phenomena of liquid surface instability subject to vertical thrust oscillation. The present study showed that CFD modeling can accurately capture all the transition physics from solid body motion, to standing surface wave, to droplet ejection at the liquid-gas interface. The present simulation also demonstrated trends consistent with experiments in the variation of physical properties, geometry, and input forcing function. It was found that thrust oscillations cause increased phase exchange area at the interface due to droplet formation, and enhanced heat transfer rate between the liquid and gas phases as the ejected droplets travel well into the warmer gas region. The first-principle based CFD simulation can capture the detailed droplet ejection as long the grid size is not larger than the droplet diameter itself.

The present effort also builds a solid foundation for the practical applications of CFD technology to address the space exploration systems involving control and management of cryogenic propellant tanks using thermodynamics vent, and to the advanced propulsion systems involving liquid jet breaks-up and atomization. 


\section{Acknowledgements}

This study was performed under a Task Order of the Jacobs Engineering NASA MSFC ESTS Contract NNM05AB50C. Dr. Ram Ramachandran was the ESTS Task Lead. Dr. Jeff West (MSFC Fluid Dynamics Branch, ER42) was the NASA Task Monitor. Authors wish to express their appreciation to Dr. John Townsend of NASA MSFC EV31 and Mr. Jeff Linder of Gray Research for helpful discussions and for sharing their experimental data used in this study. Dr. Bernard Beard of ARES Corporation provided the modal shape function in Figure 6.

\section{References}

1. M. F. Fisher, G. R. Schmidt, and J. J. Martin, "Analysis of Cryogenic Propellant Behavior in microgravity and low thrust environments", Paper presented at the 1991 Space Cryogenics Workshop, Cleveland, OH, USA, June 2, 18-20, 1991.

2. H. N. Abramson, "The Dynamic Behavior of Liquids In Moving Containers", NASA SP-106,1967.

3. CFD-ACE+, Version 2009.0, User Guide, ESI-CFD Inc., Huntsville, AL, 2009

4. H. Q. Yang, and A. J. Przekwas, "Computational Modeling of Microfluid Devices with Free Surface Liquid Handling", Technical Proceeding of the 1998 International Conference on Modeling and Simulation of Microsystems, MSM 98, pp. 498-505, 1998.

5. H. Q. Yang, J. M. Rojahn, J. W. Peugeot, D. J. Dorney. "CFD Analysis of Propellant Tank sloshing under Vertical Oscillatory Thrust and Horizontal Oscillatory Side Load", JANNAF 2008, Paper LPS-06, Feed System Design and Analysis, Orlando, November 10-13, 2008.

6. H. Q. Yang and J. W. Peugeot, "Propellant Sloshing Parameter Extraction from CFD Analysis", 46 ${ }^{\text {th }}$ AIAA/ASME/ASE/ASEE Joint Propulsion Conference \& Exhibit, July 25-28 2010, Nashville, TN. AIAA 20106889.

7. J. S. Townsend, J. Lindner, "Experimental Characterization of Axial Fluid Damping in Vibrating Cylinder Tanks", MSFC, Huntsville, AL, Private communication.

8. C. L. Goodridge, H. G. E. Hentschel, and D. P. Lathrop, "Breaking Faraday Waves: Critical Slowing of Droplet Ejection Rates", Physical Review Letters, Vol. 12, No. 15, pp. 3062-3065, 1999.

9. V. G. Levich, Physicochemical Hydrodynamics, Prentice-Hall, New Jersey, Chapter \# XI, 1962.

10. C. L. Goodridge, W. T. Shi, H. G. Hentscel, D. P. Lathrop, "Viscous Effects in Droplet-Ejecting Capillary Waves", Physical Review E, Vol. 56, No.1, pp. 472-475, 1997. 